\title{
Isometries for rank distance and permutation group of Gabidulin codes
}

\section{Thierry P. Berger}

LACO, Université de Limoges,

123 av. A. Thomas, 87060 Limoges CEDEX, FRANCE

e-mail: thierry.berger@unilim.fr tel: (33) 555457338

\begin{abstract}
The rank distance was introduced in 1985 by E. Gabidulin [1]. He determined a lower bound for the minimum rank distance of a code. Moreover, he constructed a class of codes which meet this bound: the so-called Gabidulin codes.

In this paper, we first characterize the linear isometries for the rank distance. Then we determine the isometry group and the permutation group of Gabidulin codes of full length (i.e. the length is equal to the degree of the field extension).
\end{abstract}

\section{Isometries for rank distance}

Let $K=G F\left(q^{m}\right)$ be an extension of degree $m$ of the finite field $G F(q)$. Let $E=K^{n}$ be the vector space of dimension $n$ over $K$.

Definition 1 For $a \in E, a=\left(a_{1}, \ldots, a_{n}\right)$, the rank $r k(a)$ of $a$ is the dimension of the $G F(q)$-vector space generated by $\left\{a_{1}, \ldots, a_{n}\right\}$.

Let $a$ and $b$ be two elements of $E$. The relation $d_{r}(a, b)=r k(a-b)$ defines a distance over $E$. Following this definition, it is natural to define the minimum rank distance $d_{r}$ of a code $C$. Moreover, if $d_{h}$ denotes the classical Hamming distance, then for all $a, b$ in $E$, the rank distance satisfies the inequality $d_{r}(a, b) \leq d_{h}(a, b)$.

The group of linear isometries for the classical Hamming distance is well-known: it is the monomial group of $n \times n$ matrices over $K$ with one and only one non-zero element on each row and each column [2]. This group is generated by the permutations of the support and the scalar multiplications by invertible elements on each coordinate.

In this section, we characterize the linear transformations that are isometries for the rank distance.

Definition 2 An isometry for the rank distance is a K-linear automorphism $f$ of $E$ which preserves the rank of the elements of $E$, i.e. $r k(a)=r k(f(a))$ for all a in $E$.

Let $I s o(E)$ be the group of isometries for the rank distance. The following facts are very easy to check:

- The scalar multiplications $h_{\lambda}: a=\left(a_{1}, \ldots, a_{n}\right) \mapsto \lambda a=\left(\lambda a_{1}, \ldots, \lambda a_{n}\right), \lambda \in$ $G F\left(q^{m}\right)^{*}$ are isometries for the rank distance.

- For all $M \in G L(n, q)$, the $K$-linear endomorphism $f_{M}$ of $E$ defined by $a \mapsto a M$ is an isometry for the rank distance. 
The following theorem characterizes the isometries for the rank distance.

Theorem 1 The isometry group Iso(E) for the rank distance is generated by the scalar multiplications $h_{\lambda}, \lambda \in G F\left(q^{m}\right)^{*}$ and the linear group $G L(n, q)$. This group is isomorphic to the product group $\left(G F\left(q^{m}\right)^{*} / G F(q)^{*}\right) \times G L(n, q)$.

Proof : As noticed previously, the scalar multiplications and the transformations associated to $n \times n$ invertible matrices with coefficients in $G F(q)$ are isometries for the rank distance.

Let $f \in G L\left(n, q^{m}\right)$ be an invertible $K$-linear transformation, and $M$ be its associated matrix in the canonical basis. The $i$-th row of $M$ is the image of $e_{i}$ by $f$.

Suppose that $f$ is an isometry for the rank distance. The rank of each row must be one. Moreover, eventually using a scalar multiplication, it is possible to suppose that the elements of the first row are in $G F(q)$, i.e. $f\left(e_{1}\right) \in G F(q)^{n}$.

Let $i \in\{2, \ldots, n\}$. Following the preceding remarks, there exists a $\mu \in K^{*}$ such that $\mu^{-1} f\left(e_{i}\right)$ is in $G F(q)^{n}$. This is $\mu^{-1} f_{i, j} \in G F(q)$ for all $j=1, \ldots, n$.

Let $c$ be $e_{1}+e_{i}$. The rank of $c$ is 1 . Its image is $f(c)=\left(f_{1,1}+f_{i, 1}, f_{1,2}+f_{i, 2}, \ldots, f_{1, n}+f_{i, n}\right)$ and must be of rank 1 . There exists at least one non-zero coordinate, for example the first. Set $\nu=f_{1,1}+f_{i, 1} \neq 0$. Since the rank of $f(c)$ is 1 , for a fixed $j$ there exists a $s \in G F(q)$ such that $f_{1, j}+f_{i, j}=s \nu$, i.e. $f_{1, j}+f_{i, j}=s\left(f_{1,1}+f_{i, 1}\right)$.

From this fact, we deduce $f_{1, j}-s f_{1,1}=-f_{i, j}+s f_{i, 1}$. Set $t=f_{1, j}-s f_{1,1}$. This is an element of $G F(q) \cap \mu G F(q)$. Then either $\mu$ is in $G F(q)$ and the elements of the $i$-th row are in $G F(q)$, or $t=0$, that implies $f_{1, j}=s f_{1,1}$ for all $j$ : the $i$-th row is deduced from the first by multiplication by $s$. This is not possible, since the matrix $M$ is invertible. This proves the fact that al the $f_{i, j}$ are in $G F(q)$ and $f$ is in $G L(n, q)$.

To complete the proof, we first remark that the scalar multiplications $h_{\lambda}$ commute with all the linear transformations. Moreover, the intersection of the linear group $G L(n, q)$ and the group of scalar multiplications is the subgroup of scalar multiplications for which $\lambda$ is in $G F(q)^{*}$. This implies that $I s o(E)$ is isomorphic to the direct product $\left(K^{*} / G F(q)^{*}\right) \times$ $G L(n, q)$.

\section{Gabidulin codes}

In this paragraph, we restrict ourselves to Gabidulin codes of full length, i.e. of length $n=m$, where $m$ is the degree of extension of $K=G F\left(q^{m}\right)$ over the base field $G F(q)$. The Gabidulin codes where introduced in [1]. These codes are MRD (Maximum Rank Distance): they meet the best possible rank distance $d_{r}=n+1-k$, where $k$ is the dimension of the code.

We first recall the definition and the main properties needed in the next section. Let $g=\left(g_{1}, \ldots, g_{m}\right)$ be a basis of $K$ over $G F(q)$. Let $\mathcal{G}_{k, g}$ be the matrix defined by

$$
\mathcal{G}_{k, g}=\left(\begin{array}{ccccc}
g_{1} & g_{2} & \ldots & \ldots & g_{m} \\
g_{1}^{[1]} & g_{2}^{[1]} & \ldots & \ldots & g_{m}^{[1]} \\
\vdots & \vdots & \ddots & \ddots & \vdots \\
\vdots & \vdots & \ddots & \ddots & \vdots \\
g_{1}^{[k-1]} & g_{2}^{[k-1]} & \ldots & \ldots & g_{m}^{[k-1]}
\end{array}\right)
$$


with the convention $a^{[i]}=a^{q^{i}}$.

Definition 3 The Gabidulin code of dimension $k$ relatively to the basis $g$ is the code $G_{k, g}$ of length $m$ over $K$ generated by the matrix $\mathcal{G}_{k, g}$.

In [1], E.M. Gabidulin proved that these codes are MRD.

Now, we present a more precise characterization of the dual of a Gabidulin code under their restriction that the length is exactly $m$.

Let $g=\left(g_{1}, \ldots, g_{m}\right)$ be a basis of $K$ over $G F(q)$, and $h=\left(h_{1}, \ldots, h_{m}\right)$ be the traceorthogonal basis of $g$ : $h$ is the unique basis such that $\operatorname{Tr}\left(g_{i} h_{j}\right)=\sum_{\ell=0}^{m-1} g_{i}^{[\ell]} h_{j}^{[\ell]}=\delta_{i, j}$.

Clearly, this relation is equivalent to $\mathcal{G}_{m, g} \mathcal{G}_{m, h}^{t}=I$.

Lemma 1 The dual of the Gabidulin code $G_{k, g}$ is the Gabidulin code $G_{m-k, h^{[k]}}$.

Proof : The Gabidulin code $G_{m-k, h}[k]$ is the dual of $G_{k, g}$ if and only if $<g^{[i]}, h^{[j]}>=0$ for all $i=0, \ldots, k-1$ and all $j=k, \ldots, m-1$. The result follows from the relation $\mathcal{G}_{m, h}^{t} \mathcal{G}_{m, g}=\left(<g^{[i]}, h^{[j]}>\right)_{i, j}=\mathcal{G}_{m, g} \mathcal{G}_{m, h}^{t}=I$.

Proposition 1 Suppose $k<m$. Let $G_{k, g}$ be a Gabidulin code and $h$ be the traceorthogonal basis associated to $\mathrm{g}$. A Gabidulin code $G_{m-k, h^{\prime}}$ is the dual of $G_{k, g}$ if and only if there exists a scalar $a \in K^{*}$ such that $h^{\prime}=a h$.

Proof : If the Gabidulin code $G_{m-k, h^{\prime}}$ is the dual of $G_{k, g}$ then $\left\langle g^{[i]}, h^{\prime[j]}>=0\right.$ for all $i=0, \ldots, k-1$ and all $j=0, \ldots, m-k-1$. These relations are equivalent to $<g^{[i-j]}, h^{\prime}>=0$ for all $i=0, \ldots, k-1$ and all $j=0, \ldots, m-k-1$, i.e. $<g^{[s]}, h^{\prime}>=0$ for all $s \in\left[0, m\left[\backslash\{k\}\right.\right.$. Set $a=<g^{[s]}, h^{\prime}>$.

Our conditions become $\mathcal{G}_{m, g} h^{\prime t}=a e_{k}^{t}$. The matrix $\mathcal{G}_{m, g}$ is invertible, and then the solution is unique. Moreover, $\mathcal{G}_{m, g}\left(h^{[k]}\right)^{t}=e_{k}^{t}$, and then $h^{\prime}=a h^{[k]}$. Clearly $a \neq 0$, since $h^{\prime}$ is not 0 .

The reverse part of this proposition is trivial.

From this proposition, we can deduce a characterization of the distinct bases who give the same Gabidulin code.

Theorem 2 Suppose $k<m$. Two Gabidulin codes $G_{k, g}$ and $G_{k, g^{\prime}}$ are equal if and only if if there exists a scalar $a \in K^{*}$ such that $g^{\prime}=a g$.

Proof : This result is the previous proposition applied to the dual $G_{m-k, h}$ of the Gabidulin code $G_{k, g}$.

Remark: This result cannot be directly extended to Gabidulin codes of length $n$ less than $m$.

\section{Isometry group and permutation group of Gabidulin codes}

The scalar multiplication $h_{\lambda}:\left(c_{1}, \ldots, c_{m}\right) \mapsto\left(\lambda c_{1}, \ldots, \lambda c_{m}\right), \lambda \in K^{*}$, is clearly an element of the isometry group of every $K$-linear code of $E$.

Let $M \in G L(m, q)$ be a $m \times m$ invertible matrix with coefficients in $G F(q)$ and $f \in I s o(E)$ be its associated isometry. 
Lemma 2 Let $g=\left(g_{1}, \ldots, g_{m}\right)$ be a basis of $K$ over $G F(q)$. Let $g^{\prime}=\left(g_{1}^{\prime}, \ldots, g_{m}^{\prime}\right)=g M$ be its image by $f$. The image of the Gabidulin code $G_{k, g}$ by $f$ is the Gabidulin code $G_{k, g^{\prime}}$.

Proof : Since the coefficient of $M$ are in $G F(q)$, the image of the $i$-th row $g^{[i-1]}$ by $f$ is $g^{\prime[i-1]}$. The image of the basis $\left(g^{[0]}, \ldots, g^{[k-1]}\right)$ of $G_{k, g}$ by $f$ is then the basis $\left(g^{[0]}, \ldots, g^{[[k-1]}\right)$ of $G_{k, g^{\prime}}$.

Theorem 3 Let $1 \leq k<m$. The isometry group of the Gabidulin code $G_{k, g}$ is the group of scalar multiplications, isomorphic to $K^{*}$.

Proof : The isometry group $I s o\left(G_{k, g}\right)$ of the Gabidulin code $G_{k, g}$ contains the scalar multiplications.

Reciprocally, let $f \in I s o(E)$ be an element of $I s o\left(G_{k, g}\right)$. Recall that $I s o(E)$ is generated by the scalar multiplications and the linear group $G L(m, q)$. Multiplying eventually $f$ by a scalar $\lambda$, we can suppose that $f$ is in $G L(m, k)$. Let $g^{\prime}=f(g)$. If $A$ is the matrix associated to $f$, this gives $\mathcal{G}_{m, g^{\prime}}=\mathcal{G}_{m, g} A$, and then $A=\mathcal{G}_{m, g}^{-1} \mathcal{G}_{m, g^{\prime}}$.

Moreover, using Lemma 2 and Theorem 2, there exists a scalar $a \in K^{*}$ such that $g^{\prime}=a g$. This implies $g^{\prime[i]}=a^{[i]} g^{[i]}$ for all $i=0, \ldots, m-1$. Let $A^{\prime}$ be the diagonal matrix such that $a_{i, i}^{\prime}=a^{[i]}$. We obtain $\mathcal{G}_{m, g^{\prime}}=\mathcal{G}_{m, g} A^{\prime}$, and then $A=A^{\prime}$. Recall that the elements of $A$ are in $G F(q)$, then $a^{[i]}=a$ for all $i$ and the transformation $f$ is the scalar multiplication by $a \in G F(q)^{*}$. This completes the proof.

Corollary 1 For $1 \leq k<m$, the permutation group of the Gabidulin code $G_{k, g}$ is trivial, i.e. it contains only the identity.

Proof : The permutation group is a subgroup of $I s o\left(G_{k, g}\right)$. The only scalar multiplication which is a permutation is the identity.

Conclusion In this paper, we characterized the isometries for the rank distance. Using this property, we have been able to find the permutation group and the isometry group of Gabidulin codes.

However, the problem of the classical automorphism group of Gabidulin codes for the Hamming distance remains open.

For example, if $k=1$, it is easy to construct a permutation followed by appropriated scalar multiplications on each component who leaves the code globally invariant.

We never obtained such a non-trivial example for $2 \leq k<m-1$.

\section{References}

[1] E.M. Gabidulin: Theory of codes with maximum rank distance, Problemy Peredachi Informatsii, vol.21, n.1, p. 1-12, 1985.

[2] W.C. Huffman: Groups and codes, In V.S. Pless \& W.C. Huffman Editors, Handbook of Coding Theory, Vol.II, Chapter 17, Amsterdam: Elsevier 1998. 